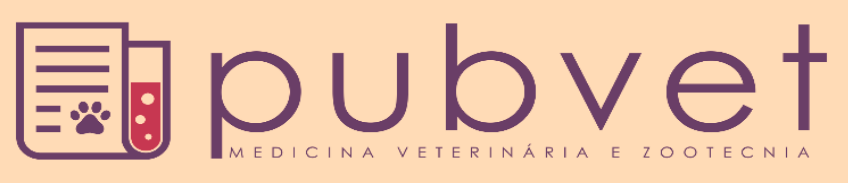

https://doi.org/10.31533/pubvet.v15n10a937.1-5

\title{
Pionefrose em cadela pós ovariohisterectomia: Relato de caso
}

\author{
Tamires Victoria Silva Natividade ${ }^{1 *} \bullet$ (D), André Marcelo Conceição Meneses ${ }^{2} \bullet$ (D) \\ ${ }^{I}$ Discente de Pós-Graduação da Universidade Federal Rural da Amazônia, Instituto Socioambiental e de Recursos Hidricos, Belém-PA-Brasil. \\ ${ }^{2}$ Professor da Universidade Federal Rural da Amazônia, Instituto da Saúde e Produção Animal, Belém - PA - Brasil. \\ *Autor para correspondência, E-mail: mvtamiresnatividade@gmail.com
}

Resumo. A pionefrose é um fenômeno caracterizado pelo preenchimento da pelve renal, cálices e ureteres por exsudato purulento em virtude de eventos obstrutivos. Sua apresentação clínica é variável e o diagnóstico tradicionalmente se dá pelos achados clínicos e ultrassonográficos. O objetivo deste trabalho é reportar um caso clínico de pionefrose pós ovariohisterectomia em cadela atendida na cidade de Belém-Pará. A paciente possuía histórico da cirurgia no ano anterior, hipofagia, apatia, distensão e dor abdominal associados à ascite, mucosas hipocoradas e aumento do tamanho dos linfonodos palpáveis. Foram solicitados hemograma, bioquímica sérica, ultrassonografia abdominal total e análise de liquido cavitário que evidenciaram efusão abdominal exsudativa e hidronefrose. Foi instituída terapia antibiótica, analgésica e hepatoprotetora e realizadas novas analises hematológicas e ultrassonografia abdominal após o período de tratamento que revelaram agravamento do quadro renal. De acordo com estes resultados a paciente foi encaminhada à nefrectomia unilateral direita e evoluiu bem clinicamente até o $4^{\circ}$ dia quando foi óbito subitamente. Este é o primeiro relato a descrever a afecção na região norte do país e além de demonstrar a importância da cuidadosa identificação das estruturas anatômicas durante o procedimento cirúrgico, também reforça a necessidade de seleção de profissional veterinário devidamente qualificado para realização do procedimento, afim de prevenir eventos pós-operatórios indesejáveis.

Palavras-chave: Cães, cirurgia, infecção

\section{Pyonephrosis following ovariohysterectomy in a bitch: Case report}

\begin{abstract}
Pyonephrosis is a phenomenon characterized by fulfillment of the kidney pelvis, calixes and ureter purulent exudate. Clinical manifestations are variable and traditionally diagnosis is given by data found in clinical examination e ultrasound findings. The aim of this paper is to report a clinical case of pyonephrosis following ovariohysterectomy in a bitch occurred in Pará state, Brazil. Patient have had surgery previously dating a year ago, hypophagia, apathy, distension and abdominal pain associated with ascites, pale mucous membranes and an increased size of palpable lymphonodes. Complete blood count, seric biochemical variables, abdominal ultrasound and analysis of cavity fluid showed exudative abdominal effusion and hydronephrosis. Antibiotics, analgesics and hepatoprotective therapy were instituted and both, new blood count analyzes and abdominal ultrasound were performed after the treatment period, which revealed worsening of renal condition. According to clinical manifestations and laboratorial results, the patient was referred to right unilateral nephrectomy and progressed well clinically until the 4th postoperative day, when she suddenly evolved to death. This is the first report to describe the condition in the northern region of the country and in addition to demonstrating the importance of careful identification of anatomical structures during the surgical procedure, it also reinforces the need to select properly a qualified veterinary professional to perform the procedure, in order to prevent undesirable postoperative events.
\end{abstract}

Key-words: Dogs, infection, surgery 


\section{Introdução}

A pionefrose é um fenômeno caracterizado pelo preenchimento da pelve renal, cálices e ureteres por exsudato purulento em virtude de eventos obstrutivos (Schmitz, 2012). O espectro clínico é variável de acordo com o tipo, severidade e local da obstrução, bem como com grau de destruição do parênquima renal e compreende desde animais assintomáticos, com inflamação de trato urinário à sépticos ou gravemente urêmicos (Chew et al., 2012; Erol et al., 2014).

O diagnóstico da enfermidade tradicionalmente se dá pelos achados clínicos e ultrassonográficos, que obedecem a premissa de extensão e localização das alterações (Geoghegan et al., 2005), requer diagnóstico diferencial de outras condições hidronefróticas para a prevenção de maiores complicações clínicas (Chan et al., 2001) e adaptação das indicações terapêuticas que incluem antibioticoterapia, nefrostomia percutânea, implante de bypass ureteral, ou em casos de pior prognóstico, a nefrectomia (Cray et al., 2018; Parry, 2005; Santarosa et al., 2007; Silva et al., 2006).

Há apenas um relato de caso descrevendo a ocorrência de pionefrose secundária à $\mathrm{OVH}$ em pequenos animais no Brasil (Apparicio et al., 2007), uma vez que a afecção tem sido mais comumente associada à litíases (Fadel et al., 2014; Maier et al., 2014). Desta maneira, o objetivo deste trabalho é reportar um caso clínico de pionefrose pós OVH em cadela atendida na cidade de Belém-Pará.

\section{Relato do caso}

Foi atendido em domicílio um canino, fêmea, cinco anos de idade, sem raça definida, pesando nove $\mathrm{kg}$, cujos tutores relataram histórico histerectomia no ano anterior, realizada em estabelecimento irregular por indivíduo não detentor de registro no conselho regional de medicina veterinária do Estado do Pará. Ao exame físico a paciente apresentava escore corporal ruim, histórico de apatia e ectoparasitas, dor abdominal associado à ascite, mucosas hipocoradas, temperatura retal de $39^{\circ} \mathrm{C}$, aumento de tamanho dos linfonodos mandibular, pré-escapular e inguinal.

Após avaliação clínica foram realizadas coletas de amostras sanguíneas para hemograma e bioquímica sérica (ureia, creatinina, alanina aminotransferase e fosfatasealcalina), abdominocentese para coleta e análise de líquido cavitário e ultrassonografia abdominal total. O hemograma revelou leucocitose $\left(41,100 / \mathrm{mm}^{3}\right)$ e anemia normocítica normocrômica importante (He: $3,7 \mathrm{milhões} / \mathrm{mm}^{3} ; \mathrm{Hb}$ : 8,2g/dl; Ht: 26\%), a despeito dos valores de referência (leucócitos: $6.000-17.000 / \mathrm{mm}^{3}$; He: 5,5-8,5 milhões/mm³; Hb: 12-18g/dL; Ht:35-55\%) (Jain \& Jain, 1993).

A bioquímica sérica não demonstrou anormalidades de função renal, havendo, porém, elevação das concentrações séricas alanina aminotransferase (ALT) e fosfatase alcalina (FA), cujos valores foram respectivamente 123UI/dL e 807UI/dL (ALT: 21-86UI/L; FA: 20-156UI/L) (Kaneko et al., 2008). A avaliação de líquido cavitário revelou fluido de aspecto leitoso, turvo, com leucócitos abundantes e predomínio de polimorfonucleares, características citológicas compatíveis com efusão abdominal exsudativa (Valenciano \& Cowell, 2019).

Os achados ultrassonográficos evidenciavam hepatoesplenomegalia, presença de líquido abdominal livre e hidronefrose unilateral direita, cujo órgão apresentava, em média, dimensões 2,5x maiores que a esperada para a mesma faixa de peso corporal (> 12,1 cm de eixo longitudinal) (Sampaio \& Araújo, 2002), região cortical adelgaçada e destruição do parênquima por acúmulo de conteúdo anecóico heterogêneo, indicativo de hidronefrose (Figura 1A). Mediante ao quadro clínico e achados dos exames complementares foi confirmado o diagnóstico de pionefrose unilateral direita e instituída terapia com enrofloxacino ( $5 \mathrm{mg} / \mathrm{kg} / \mathrm{uma}$ vez ao dia - SID), metronidazol $(15 \mathrm{mg} / \mathrm{kg} / \mathrm{duas}$ vezes ao dia - BID), omeprazol ( $1 \mathrm{mg} / \mathrm{kg} / \mathrm{SID})$ e silimarina $(20 \mathrm{mg} / \mathrm{kg} / \mathrm{SID})$ por 30 dias, além de dipirona $(25 \mathrm{mg} / \mathrm{kg} / \mathrm{BID})$ por cinco dias.

Após o período de tratamento prescrito foram realizadas novas análises hematológicas e ultrassonografia abdominal que demonstraram redução da leucocitose $\left(22,000 \mathrm{~mm} / \mathrm{mm}^{3}\right)$ e anemia importante (HE: 3,7milhões $/ \mathrm{mm}^{3}$; HB: 7,6g/dl; HT: 25\%) e FA 348,0 UI/L. O exame de imagem revelou mesentério reativo e acentuada coleção líquida encapsulada de cavitações comunicantes $(>14 \mathrm{~cm}$ de eixo longitudinal), deslocando estruturas subjacentes, com abundantes pontos ecoicos em suspensão (igura $\underline{1 \mathrm{~B})}$. 
De acordo com a evolução do quadro clínico e achados ultrassonográficos a paciente foi encaminhada à nefrectomia unilateral direita. Após remoção cirúrgica do rim foi possível observar acentuado aumento de tamanho devido à acumulo de exsudato, não sendo possível distinguir o ureter, com nítida perda de contorno anatômico, além de estrutura rígida aderida ao mesmo, semelhante ao lacre cirúrgico utilizado para realização de OVH (Figura 2).
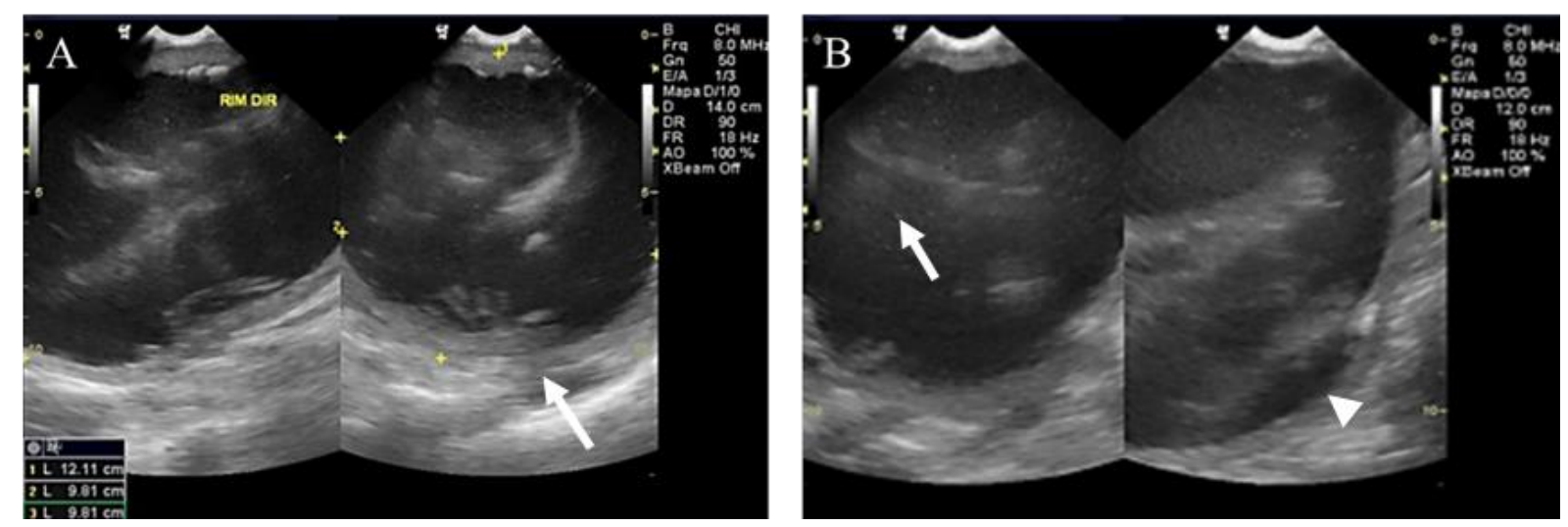

Figura 1. A. Imagens ultrassonográficas evidenciando rim direito com dimensões acentuadamente aumentadas (seta branca). B. Imagem ultrassonográfica revelando estrutura renal ainda mais alterada com cápsula renal adelgaçada (cabeça de seta) e com pontos ecoicos em suspensão (seta branca), quadro compatível com pionefrose.

No pós-operatório foi instituída fluidoterapia com ringer com lactato ( $40 \mathrm{ml} / \mathrm{kg} / 24 \mathrm{~h})$, ceftriaxona ( 25 $\mathrm{mg} / \mathrm{kg} / \mathrm{BID})$, omeprazol $(0,7 \mathrm{mg} / \mathrm{kg} / \mathrm{SID})$, tramadol (2 $\mathrm{mg} / \mathrm{kg} / \mathrm{BID})$, morfina $(0,3 \mathrm{mg} / \mathrm{kg} / \mathrm{TID})$, meloxicam $(0,1 \mathrm{mg} / \mathrm{kg} / \mathrm{SID})$ dipirona $(25 \mathrm{mg} / \mathrm{kg} / \mathrm{BID})$ e silimarina $(20 \mathrm{mg} / \mathrm{kg} / \mathrm{SID})$. A paciente evoluiu sem complicações clínicas até o $4^{\circ}$ dia de pós-operatório, quando foi a óbito subitamente.

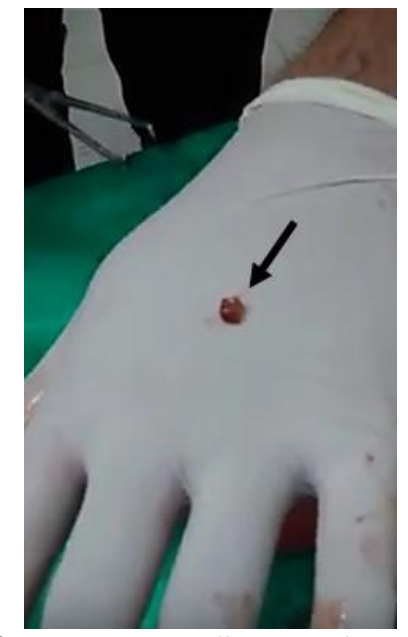

Figura 2. Estrutura semelhante ao lacre cirúrgico (seta) encontrada aderida ao rim direito após

\section{Discussão} nefrectomia.

O diagnóstico de eventos hidronefróticos pode ser complexo, tendo em vista a pluralidade de sintomas e requer minuciosa investigação pregressa e avaliação física, bem como exames complementares que sustentem a hipótese diagnóstica. No presente relato, os achados evidenciam quadro de pionefrose unilateral direta, de acordo com as imagens ultrassonográficas compatíveis com renomegalia e presença de conteúdo hiperecóico em suspensão, consistente com os achados de Choi et al. $(\underline{2010})$.

Alguns autores já investigaram a ocorrência de complicações pós-operatórias em cadelas submetidas à ovário-histerectomia, cuja incidência é variável de acordo com a habilidade do cirurgião. Estes estudos comprovaram que alterações urológicas são infrequentes e podem estar associadas excisão ou ligadura acidental do ureter, granuloma de coto uterino aderido à vesícula urinária ou aderência ureteral ao corno 
uterino (Apparicio et al., 2007; Atallah et al., 2013; Fossum, 2014; Kyles et al., 1996). Neste caso, foi observado o clampeamento do ureter por lacre cirúrgico, impedindo o fluxo urinário adequado e originando dilatação de pelve renal, com posterior infecção e destruição progressiva do parênquima.

Similar ao observado por Choi et al. (2010), nos exames hematológicos da paciente em questão também foi constatada leucocitose em ambos os hemogramas realizados antes e após a terapia conservadora. As alterações de concentrações de enzimas hepáticas e anemia encontradas na paciente deste já foram reportadas em pacientes humanos e veterinários com diferentes graus de comprometimento do estado geral (Kyles et al., 1996; Silva et al., 2006).

Não foi observada azotemia na paciente deste estudo, sendo este um achado variável, pois que seu aparecimento está sujeito à distribuição da enfermidade (uni ou bilateral), grau de destruição e consequente perda de função, desta maneira, já foram relatados anteriormente casos de pionefrose unilateral em humanos e animais sem a ocorrência simultânea de azotemia (Chew et al., 2012; Kyles et al., 1996; Silva et al., 2006).

O manejo clínico das infecções de trato urinário superior prevê a utilização de antibióticos de amploespectro por até 6 semanas (Weese et al., 2019), já as abordagens cirúrgicas mais invasivas estão reservadas à quadros severos e incluem nefrostomia percutânea ecoguiada, utilização dispositivos bypass, ou, em casos de pequena possibilidade de manutenção funcional do órgão, a nefrectomia (Cray et al., 2018; Fossum, 2014; Santarosa et al., 2007). Visto que a grave renomegalia deste relato não apresentou evolução satisfatória mediante o tratamento clínico conservador, foi indicada nefrectomia unilateral direita.

Segundo Choi et al. (2010), o prognóstico da enfermidade varia de bom à reservado, porém, está condicionado à rapidez de identificação e início do tratamento. No entanto, a paciente em estudo evoluiu clinicamente bem até o $4^{\circ}$ dia de pós-operatório quando foi a óbito, possivelmente em consequência da evolução insidiosa da enfermidade e decorrente atraso diagnóstico.

\section{Conclusões}

A pionefrose é considerada uma condição clínica severa e requer intervenção clínico-cirúrgica precoce. Este é o primeiro relato a descrever a afecção na região norte do país e além de demonstrar a importância da cuidadosa identificação das estruturas anatômicas durante o procedimento cirúrgico, também reforça a necessidade de seleção de profissional veterinário devidamente qualificado para realização do procedimento, afim de prevenir eventos pós-operatórios indesejáveis.

\section{Referências bibliográficas}

Apparicio, M., Vicente, W. R. R., Farias, A., Covizzi, G. J., Ribeiro, A. P. C., \& Gadelha, C. R. F. (2007). Pyonephrosis following ovariohysterectomy in a bitch: case report. Ars Veterinaria, 23(1), 19-22. https://doi.org/10.15361/2175-0106.2007v23n1p19-22.

Atallah, F. A., Silva, R. S., Ramos, M. L. M., Oliveira, A. L. A., França, T. N., \& Brito, M. F. (2013). Complicações pós-cirúrgicas em cadelas submetidas a ovário-histerectomia no Rio de Janeiro. Brazilian Journal of Veterinary Medicine, 35(Supl. 1), 61-69. https://doi.org/http://rbmv.org/index.php/BJVM/article/view/648/508.

Chan, J. H. M., Tsui, E. Y. K., Luk, S. H., Fung, S. L., Cheung, Y. K., Chan, M. S. M., Yuen, M. K., Mak, S. F., \& Wong, K. P. C. (2001). MR diffusion-weighted imaging of kidney: differentiation between hydronephrosis and pyonephrosis. Clinical Imaging, 25(2), 110-113. https://doi.org/10.1016/S0899-7071(01)00246-7.

Chew, D. J., Dibartola, S. P., \& Schnck, P. A. (2012). Urologia e nefrologia do cão e do gato. SaundersEnsevier.

Choi, J., Jang, J., Choi, H., Kim, H., \& Yoon, J. (2010). Ultrasonographic features of pyonephrosis in dogs. Veterinary Radiology \& Ultrasound, 51(5), 548-553. https://doi.org/10.1111/j.17408261.2010.01702.x.

Cray, M., Berent, A. C., Weisse, C. W., \& Bagley, D. (2018). Treatment of pyonephrosis with a subcutaneous ureteral bypass device in four cats. Journal of the American Veterinary Medical 
Association, 252(6), 744-753. https://doi.org/10.2460/javma.252.6.744.

Erol, A., Çoban, S., \& Tekin, A. (2014). A giant case of pyonephrosis resulting from nephrolithiasis. Case Reports in Urology, 2014, 1-3. https://doi.org/10.1155/2014/161640.

Fadel, L., Bluwol, K., \& da Silva, D. R. (2014). Pionefrose associada a nefrólitos em um cão-relato de caso. Revista Científica de Medicina Veterinária, 176-180.

Fossum, T. W. (2014). Cirurgia de pequenos animais (4th ed., Vol. 1). Elsevier Brasil.

Geoghegan, T., Govender, P., \& Torreggiani, W. C. (2005). MR urography depiction of fluid-debris levels: A sign of pyonephrosis. American Journal of Roentgenology, 185(2), 560. https://doi.org/10.2214/ajr.185.2.01850560.

Jain, N. C., \& Jain, A. H. (1993). Essentials of Veterinary Hematology (1st ed.). Wiley-Blackwell.

Kaneko, J. J., Harvey, J. W., \& Bruss, M. L. (2008). Clinical biochemistry of domestic animals (6th ed. (ed.); Vol. 1). Academic press.

Kyles, A. E., Douglass, J. P., \& Rottman, J. B. (1996). Pyelonephritis following inadvertent excision of the ureter during ovariohysterectomy in a bitch. The Veterinary Record, 139(19), 471-472. https://doi.org/10.1136/vr.139.19.471.

Maier, R., Lutter, F. X., \& Lohss-Baumgärtner, E. (2014). Pyonephrose durch Xanthinsteine bei einer mit Allopurinol behandelten Hündin. Tierärztliche Praxis Ausgabe K: Kleintiere/Heimtiere, 42(1), 49-54. https://doi.org/10.1055/s-0038-1623742.

Parry, N. M. A. (2005). Pyelonephritis in small animals. UK Vet, 10(6), 1-5.

Sampaio, K. M. O. R., \& Araújo, R. B. (2002). Ultrasonographic measurement of linear characteristics and renal volumetry in dogs. Arquivo Brasileiro de Medicina Veterinária e Zootecnia, 54(3), 248254. https://doi.org/10.1590/s0102-09352002000300005.

Santarosa, I. A. M., Godoy, C. L. B., Pippi, N. L., Antunes, P. S. P., Rappeti, J. C. S., Krolikowski, G., Novosad, D., \& Gheller, V. S. (2007). Nefrostomia percutânea ecoguiada em cães. Ciência Rural, 37(3), 762-768. https://doi.org/10.1590/s0103-84782007000300025.

Schmitz, P. . (2012). Rins: uma abordagem integrada à doença. Artmed.

Silva, S. L., Silva Júnior, G., Gomes, J. S., Campos, H. H., \& Daher, E. F. (2006). Pionefrose em Paciente com Cálculo Ureteral Infectado sem Comprometimento do Estado Geral. Jornal Brasileiro de Nefrologia, 28(4), 213-217.

Valenciano, A. C., \& Cowell, R. L. (2019). Cowell and Tyler's diagnostic cytology and hematology of the dog and cat-E-Book. Elsevier Health Sciences. https://doi.org/DOI: 10.1016/c2016-0-02017-x.

Weese, J. S., Blondeau, J., Boothe, D., Guardabassi, L. G., Gumley, N., Papich, M., Jessen, L. R., Lappin, M., Rankin, S., \& Westropp, J. L. (2019). International Society for Companion Animal Infectious Diseases (ISCAID) guidelines for the diagnosis and management of bacterial urinary tract infections in $\operatorname{dogs}$ and cats. The Veterinary Journal, 247, 8-25. https://doi.org/10.1016/j.tvj1.2019.02.008.

Histórico do artigo:

Recebido: 2 de maio de 2021.

Aprovado: 29 de junho de 2021.
Licenciamento: Este artigo é publicado na modalidade Acesso Aberto sob a licença Creative Commons Atribuição 4.0 (CC-BY 4.0), a qual permite uso irrestrito, distribuição, reprodução em qualquer meio, desde que o autor e a fonte sejam devidamente creditado. 